\title{
Acute Segmental Testicular Infarction caused by COVID-19 Disease: A Case Report and Current Literature Review
}

\author{
(1) Murat Demir*, (๑llyas Dundar** \\ *Van Yuzuncu Yil University Faculty of Medicine, Department of Urology, Van, Turkey \\ **Van Yuzuncu Yil University Faculty of Medicine, Department of Radiology, Van, Turkey
}

Abstract

Coronavirus disease-2019 (COVID-19) is known to cause sepsis-associated hypercoagulopathy. Thromboembolic complications affecting many systems associated with COVID-19 disease have been described in the literature. The direct effect of this virus on male urogenital organs and possible testicular damage is still being evaluated. Follow-up studies in recovering male patients are necessary to investigate the possibility of testicular damage. In this article, we aimed to present a 30-year-old patient who presented with acute testicular pain and was diagnosed with testicular infarction due to COVID-19 with ultrasonography and contrast-enhanced magnetic resonance imaging findings.

Keywords: COVID-19, coronavirus disease, testicolar infarction, ultrasonography, magnetic resonance inaging

\section{Introduction}

Although the most frequently affected system is the respiratory system in Coronavirus disease-2019 (COVID-19), this infection leads to symptoms in other systems as well. Moreover, it affects other systems mostly due to hypercoagulation, and although most thrombotic events related to COVID-19 are associated with deep vein thrombosis, complications related to arterial structures have also been reported (1). On the other hand, although COVID-19 has been shown to act through angiotensin converting enzyme (ACE) receptors and disrupt spermatogenesis in the testicles, to our knowledge, there has been no report of testicular infarction associated with COVID-19 in the literature (2). In this report, we present a case of segmental testicular infarction caused by COVID-19.

\section{Case Report}

A consent form was obtained from the patient for this case report. The 30-year-old male patient presented to our urology clinic with a two-week history of pain in the left testicle. The patient had no history of orchitis, vasculitis, polycythemia, or other comorbidities, and no history of trauma. The patient's complaint started as an acute, severe pain on the $4^{\text {th }}$ day of COVID-19 treatment, and he recovered from COVID-19 two weeks earlier, which had been confirmed by polymerase chain reaction. It was also revealed that the patient discontinued his favipiravir therapy that was initiated for COVID-19 treatment and used no anticoagulant drug throughout the infection. Physical examination was unremarkable except for left testicular tenderness. Complete blood and biochemistry tests were normal, and complete urinalysis, brucella, acidresistant bacilli in urine, tuberculosis, and culture tests were negative. Scrotal doppler ultrasonography (US) that was performed due to the presence of varicocele four weeks earlier was also unremarkable, whereas it showed a hypoechoic area with geographic borders without vascular flow in the subcapsular region at the inferior pole of the left testicle, which was approximately $7 \times 6$ $\mathrm{mm}$ in size and was suggestive of an infarction (Figure 1). Contrast-enhanced magnetic resonance imaging (MRI) showed increased signal intensity on T2-weighted images (T2-WI) and showed no contrast enhancement 


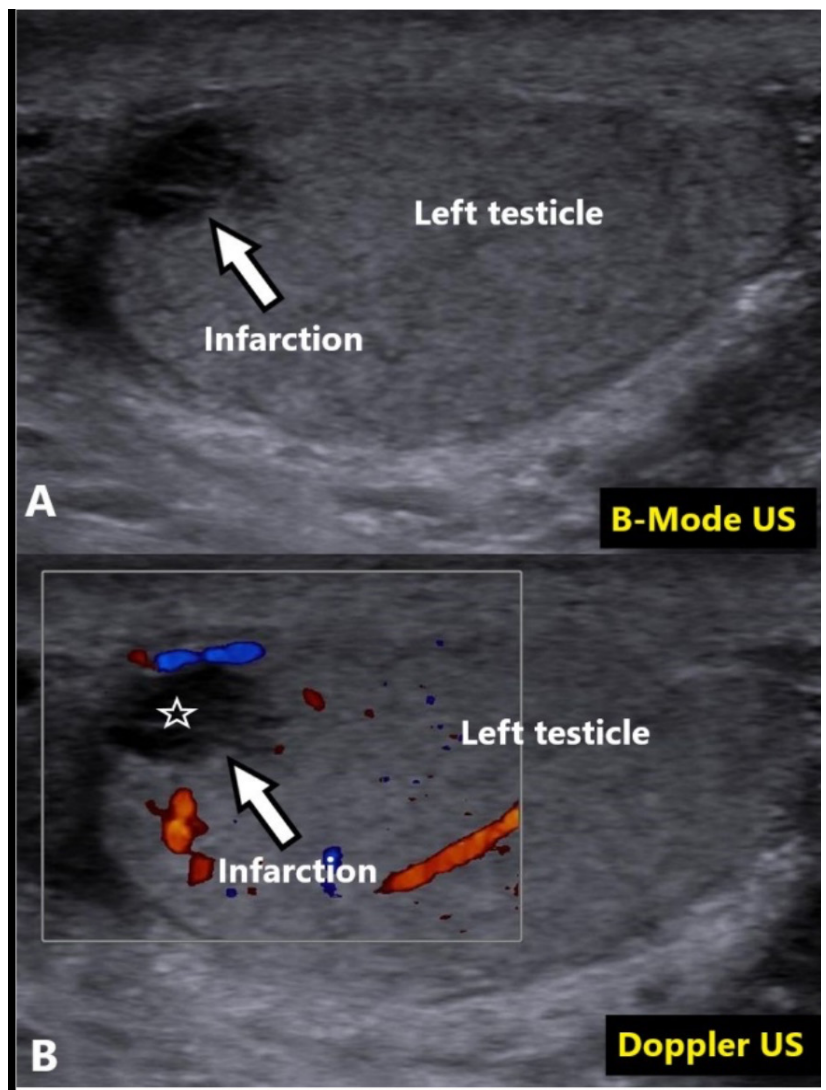

Figure 1. Scrotal US showing a wedge-shaped hypoechoic area with geographic borders, approximately $7 \times 6 \mathrm{~mm}$ in size, in the subcapsular region at the inferior pole of the left testicle in $B$-mode images. ( $A$, arrow). There was no vascularity (star) in this area on doppler US (B, arrow)

US: Ultrasonography

on T1-weighted images (T1-WI) in the region detected by the US (Figure 2). These radiological findings were consistent with a segmental testicular infarction. An anticoagulant was given to the patient as treatment. The patient did not continue to follow-up.

\section{Discussion}

Segmental testicular infarction is a type of ischemia caused by arterial occlusion of a focal area in the testicle, mostly presenting with acute pain (3). It is highly difficult to distinguish this entity from other acute testicular pathologies. Common etiologies include trauma, orchitis, polycythemia, vasculitis, and diseases that cause hypercoagulation. In most cases, the underlying cause cannot be determined, while infectious events are the most commonly blamed. Color doppler US is the primary imaging method, with the most common findings including rounded or wedge-shaped intra-testicular hypoechoic lesions with decreased or completely disappearing vascularity (4).

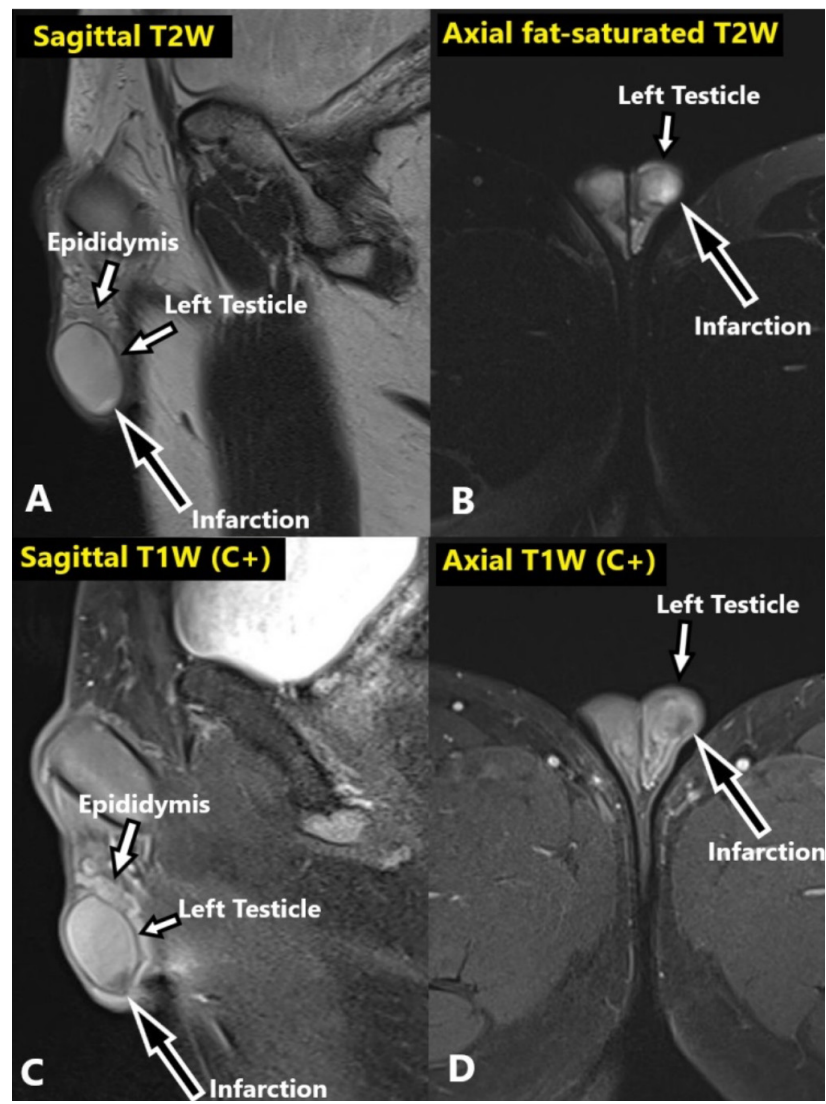

Figure 2. Non-fat-saturated sagittal (A) and fat-saturated axial (B) T2-weighted MRI showing an increased wedge-shaped signal intensity in the subcapsular region at the inferior pole of the left testicle (arrows). Contrast-enhanced sagittal (C) and axial (D) T1weighted MRI showing no contrast enhancement in that area MRI: Magnetic resonance imaging

In patients that cannot be diagnosed definitively on doppler US, the diagnosis can be supported by MRI since it can easily visualize the borders of segmental testicular infarction not only on T2-WI but also on contrastenhanced images. In segmental testicular infarction, the signal intensity of T1-T2-WI varies depending on the age of the infarction (5). Although T2-WI shows well-defined borders and mostly shows lower signal intensity (66\%), they may also show a high (17\%) or intermediate signal $(17 \%)$ intensity (3). In our case, T2-WI showed high signal intensity in the infarction area. In our case, we think that this high signal intensity is related to the infarction age in the chronic period. The infarction area shows no contrast enhancement on contrast-enhanced T1-WI $(3,6)$. In our case, there was no contrast enhancement consistent with the literature.

COVID-19 has been shown to have several effects on testicles, such as necrosis and apoptosis in spermatogenic cells via inflammatory infiltration. Autopsies performed after COVID-19 showed that ACE receptor expression and spermatogenesis were 
impaired in the testis (7). In addition to these effects, the present study detected another effect of COVID-19 on testicles, i.e. infarction associated with testicular arterial embolism, which, to our knowledge, has never been reported in the literature.

Infections in the body trigger inflammation, thereby leading to an increase in inflammatory elements, ultimately activating the coagulation system. This cascade system is known as thromboinflammation, which is a complement system involving microorganism-derived products, polyphosphate, free DNA fragments, and mast cells. In turn, these triggering events cause increased D-dimer and interleukin- 6 levels, thereby leading to an increased risk of hypercoagulative complications $(8,9)$. Although hypercoagulative complications associated with COVID-19 mostly occur in deep veins, complications associated with arterial structures have also been reported in the literature, some of which include cardiac embolism and retinal artery occlusion due to COVID-19 (10).

In conclusion, utmost care should be taken for the development of testicular arterial embolism in COVID-19 patients presenting with testicular pain.

\section{Ethics}

Informed Consent: A consent form was obtained from the patient for this case report.

\section{Authorship Contributions}

Concept: M.D., I.D., Design: M.D., Data Collection or Processing: M.D., I.D., Analysis or Interpretation: M.D., I.D., Literature Research: M.D., I.D., Writing: M.D., I.D.

Conflict of Interest: No conflict of interest was declared by the authors.

Financial Disclosure: The authors declared that this study received no financial support.

\section{References}

1- Yilmaz A, Zerde HC, Alay B, Ergul Z. Effect of COVID-19 Pandemic on the Working of Blood Transfusion Center: A CrossSectional Study. Med Bull Haseki 2021;59(Suppl 1):15-20.

2- Younis JS, Abassi Z, Skorecki K. Is there an impact of the COVID-19 pandemic on male fertility? The ACE2 connection. Am J Physiol Endocrinol Metab 2020;318:878-80.

3- Fernández-Pérez GC, Tardáguila FM, Velasco $M$, et al. Radiologic findings of segmental testicular infarction. Am J Roentgenol 2005;184:1587-93.

4- Madaan S, Joniau S, Klockaerts K, et al. Segmental testicular infarction: conservative management is feasible and safe. Eur Urol 2008;53:441-5.

5- Sweet $D E$, Feldman MK, Remer EM. Imaging of the acute scrotum: keys to a rapid diagnosis of acute scrotal disorders. Abdom Radiol (NY) 2020;45:2063-81.

6- Smets T, Reichman G, Michielsen DPJ. Segmental testicular infarction: a case report. J Med Case Rep 2017;11:140.

7- Achua JK, Chu KY, Ibrahim E, et al. Histopathology and ultrastructural findings of fatal COVID-19 infections on testis. World J Mens Health 2021;39:65-78.

8- Connors JM, Levy JH. COVID-19 and its implications for thrombosis and anticoagulation. Blood 2020;135:2033-40.

9- Ranucci M, Ballotta A, Di Dedda $U$, et al. The procoagulant pattern of patients with COVID-19 acute respiratory distress syndrome. J Thromb Haemost 2020;18:1747-51.

10- Shantha JG, Auld SC, Anthony C, et al. Retinopathy and Systemic Disease Morbidity in Severe COVID-19. Ocul Immunol Inflamm 2021;29:743-50. 